\title{
The role of surrogate models in combined aeroelastic and structural optimization of cable-stayed bridges with single box deck
}

\author{
M. Cid Montoya, S. Hernández, I. Kusano, F. Nieto \& J. Á. Jurado \\ Structural Mechanics Group, School of Civil Engineering, \\ Universidade da Coruña, Spain
}

\begin{abstract}
The aeroelastic phenomena represent a crucial issue in the design of long span bridges and their relevance grows as the length of the spans is increased, which is the current trend in these mega-structures. The search for the best performing deck cross-section, as well as the search of the most economic bridge design, are two of the most relevant goals for bridge designers. Hence, the pursuit of good aerodynamic performance of the bridge deck cross-section and the structural optimization of the bridge are destined to converge in a unified process to achieve more efficient bridge designs, given their interdependence. This paper presents an integrated methodology based on a surrogate-based optimization process to obtain an optimum bridge design considering structural and aeroelastic constraints. The deck cross-section used in this work is the well-known G1 section, in which two deck shape variables are considered. The aerodynamic behavior of the cross sections analyzed by the optimization algorithm is given by a surrogate model trained with a set of designs calculated with CFD techniques. The structural performance is analyzed by means of finite element analysis. The areas and prestressing forces of the stays and the deck shape and plate thickness are considered as design variables, and the optimization of the bridge is carried out by a gradient-based optimization algorithm.

Keywords: structural optimization, bridge aerodynamics, cable stayed bridges, CFD, flutter analysis.
\end{abstract}




\section{Introduction}

The main challenge in the bridge engineering field is the increasing span length of the new bridge projects that are currently built around the world. This trend involves taking into account the aeroelastic phenomena as some of the most relevant design constraints, keeping in mind of the structural behavior of the bridge.

The aeroelastic behavior of a bridge is mainly driven by two capital aspects, the dynamic response of the bridge and the aeroelastic properties of the deck crosssection. The relative relevance of these two factors in the aeroelastic responses is influenced by the length of the main span of the bridge, which is the main cause that determines the global stiffness of the structure. While in bridges with moderate span length the aeroelastic phenomena can be controlled by the stiffness of the bridge, in cases with longer spans, the global stiffness of the structure becomes insufficient, and the only way to avoid undesirable wind induced effects is to modify the deck cross-section. It must be noticed that the interdependence of these two aspect has to be considered, given that modifying the deck crosssection affects to its structural stiffness. Besides, both aspects present conflicting tendencies, given that good aeroelastic performance of deck cross-sections implies slender geometries, which reduces the mechanical properties of the section.

The influence of the deck cross-section shape on the aeroelastic response of a cable suspension bridge is well known since many years ago. Several researchers have written reports where the influence on the aerodynamic responses due to shape modifications is studied, as is the case for instance of [1]. However, heuristic approaches to improve bridge design considering only deck shape changes can not reach the optimum, as they disregard other considerations that also influence the solution such as structural responses. This is the main reason that justifies the use of methodologies that consider both aspects simultaneously, as it is the case of optimization process with both types of constraints. One consequence of this approach is the need for obtaining all the results required computationally in every step of the bridge design.

The use of optimization algorithms in structural design is currently a widespread technique, and some insights about this field can be found for instance, in $[2,3]$. The application of structural optimization is also widely employed, and an interesting application to optimize the cross-section of the cables of a cablestayed bridge can be found in [4]. In [5], optimization algorithms were used to optimize the deck size and the main cable cross-section area of the Messina Bridge project in Italy. However, deck shape modifications were not considered yet. Some preliminary results of optimization of stays and deck shape and size in a cable stayed bridge considering a set of semicircular cross-sections defined with one shape design variable have been reported in [6]. The aerodynamic coefficients $C_{L}$, $C_{M}$ and $C_{D}$ were obtained for a set of values of the design variables and then fitted to a smooth spline, which allowed to predict the aerodynamic coefficients of the designs proposed by the optimization algorithm. However, deck girders of real bridges present complex shapes and they can be modified by means of several 


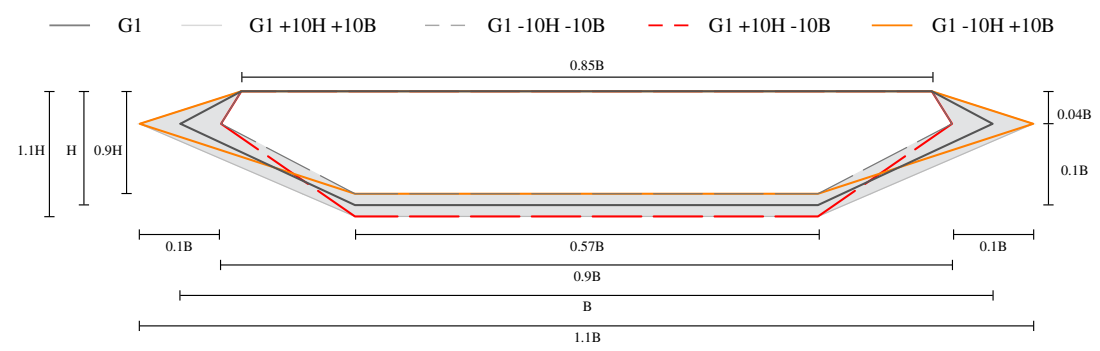

Figure 1: Definition of the considered design domain.

shape changes, giving place to more complicated problems. This work present the definition of the optimization problem for obtaining a more realistic deck shape (see figure 1) including more shape design variables in the definition of the cross section.

The section studied in this work is the well-known G1 section, as described in [7]. The chosen domain is defined by percentage variations of the depth $H$ and width $B$ of the G1 section, which is considered the base or initial design, as it can be seen in figure 1. The variations are up to a percentage of $10 \%$ for both dimensions, which modifies the geometrical properties of the section in the quantities indicated in table 1 . The modification in the dimensions involves changes in the depth of $0.84 \mathrm{~m}$ and $6 \mathrm{~m}$ in the width, which means a variation on the dimensions ratio from 5.84 to 8.73 . The angles reported in table 1 describe the total angle between the inclined sides of the deck, and the top and bottom angles are measured with respect to the horizontal direction. The relationship between the top and bottom perimeter is also reported. The aerodynamic coefficients of the cross-sections of the deck will be obtained for a set of values of the design

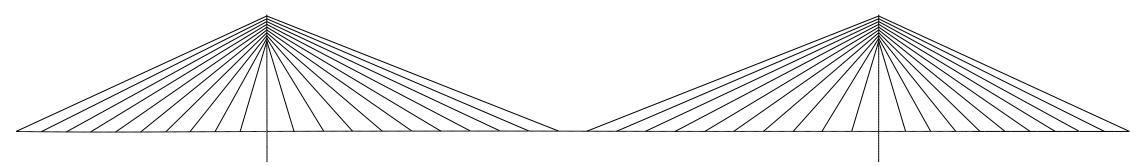

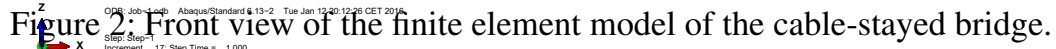
increment 17 : Step Time $=1.000$

The bridge proposed to test the methodologies presented in this work is a project of a cable-stayed bridge designed in La Coruña, Spain, with a main span length of $658 \mathrm{~m}$ and two lateral spans of $270 \mathrm{~m}$. The finite element model is shown in figure 2 


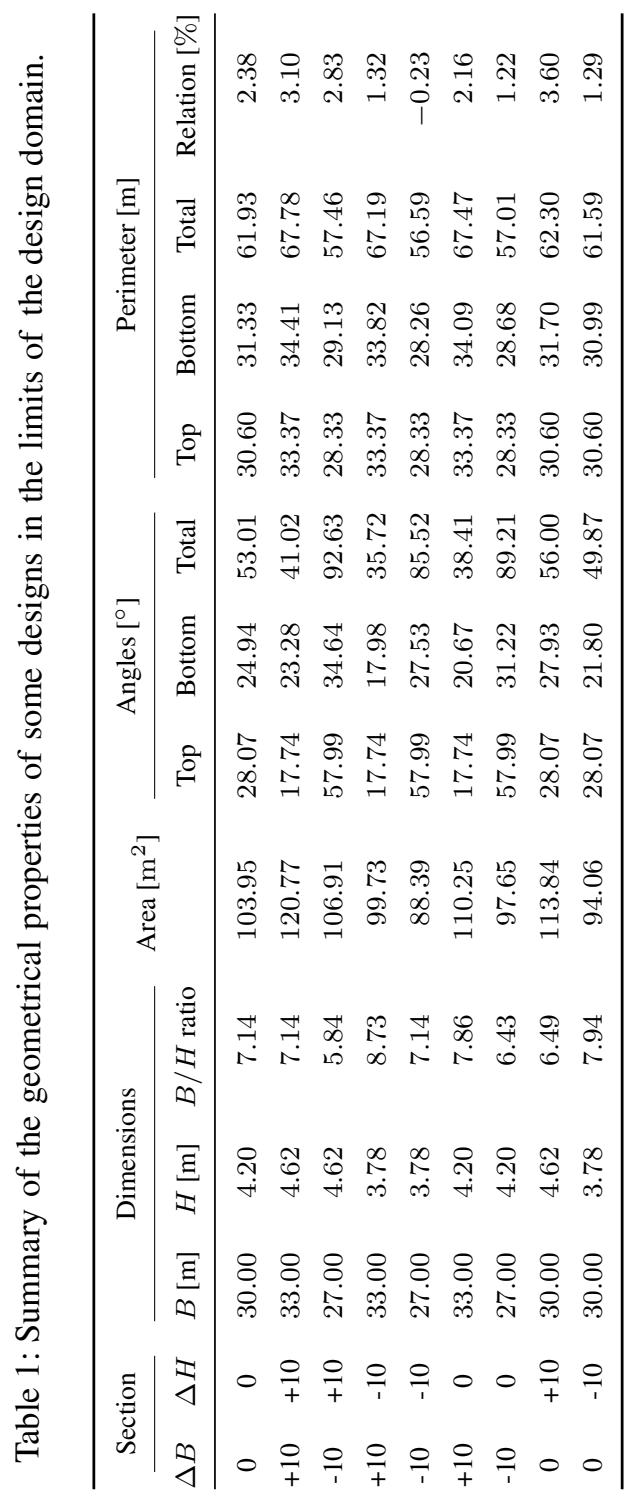


and consists of bar elements for the deck and towers and truss elements to simulate the stays. The simulation code used to analyze the model was Abaqus/ Standard 6.13 [9] software.

This work presents some improvements in the methodology developed in [6] to make it more general, particularly in the aeroelastic characterization process. In the following sections, the process for obtaining the aeroelastic properties of each design proposed by the optimization algorithm is described, which is based on the use of surrogate models, and some results are presented. Later, the optimization approach is also described and commented.

\section{Aeroelastic characterization of a single box deck}

Given that in the optimization process described before some of the design variables are shape parameters of the deck cross-section, the aeroelastic behavior of each section proposed by the algorithm has to be characterized. As the crosssections considered in the design domain can be accepted as streamlined shapes, the quasi-steady theory formulation [10] can be applied to obtain the flutter derivatives from the aerodynamic coefficients of each shape. Therefore, under this assumption, the only required task is to obtain these aerodynamic coefficients. This can be done computationally by means of Computational Fluid Dynamics (CFD) techniques (see for instance $[11,12]$ ) for a set of designs and afterwards applying surrogate models [8] to generate these coefficients for every section required during the optimization process as described below.

\subsection{Computational evaluation of aerodynamic coefficients}

In order to build the surrogate model, some designs for selected values of $B$ and $H$ of the design domain have to be evaluated at two angles of attack $\alpha=\left[0^{\circ}, 2^{\circ}\right]$ to obtain the values and slopes of the aerodynamic coefficients, as required by the quasi-steady theory. These coefficients are given by

$$
C_{L}=\frac{L}{\frac{1}{2} \rho U^{2} B}, \quad C_{D}=\frac{D}{\frac{1}{2} \rho U^{2} B} \quad \text { and } \quad C_{M}=\frac{M}{\frac{1}{2} \rho U^{2} B^{2}},
$$

where $L, D$ and $M$ are the lift and drag forces and moment, respectively, considering the sign convention indicated in figure 3, $\rho$ is the air density, $U$ is the wind velocity and $B$ is the width of the deck cross-section.

The CFD analyses were conducted in the open source code OpenFOAM [13] using a 2D unsteady Reynolds-averaged Navier-Stokes (URANS) approach. The turbulence model considered was Menter's SST $k-\omega$ model, first developed in [14] and improved in [15].

The meshes created have a number of elements of about $2.8 \cdot 10^{5}$, and details of two of them can be seen in figures 5 and 6 corresponding to the designs $+10 \% \mathrm{H}$ $+10 \% \mathrm{~B}$ and $+10 \% \mathrm{H}-10 \% \mathrm{~B}$, respectively. They are structured meshes and were generated by the in-house software FLUSINI [16]. The analyses were carried out 


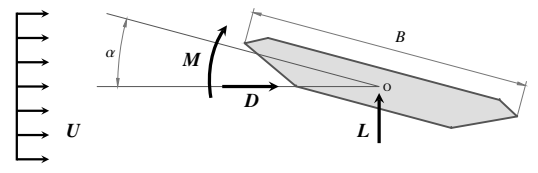

Figure 3: Sign convention for the aerodynamic forces.

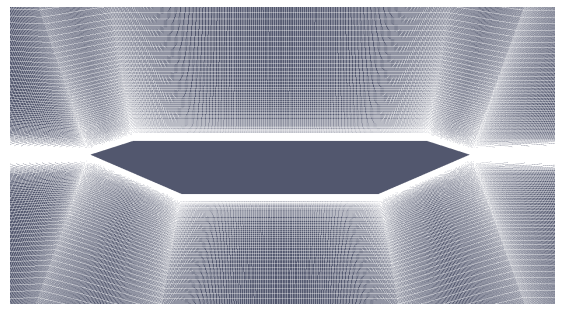

Figure 5: Detail of the mesh of the section $+10 \% \mathrm{H}+10 \% \mathrm{~B}$.

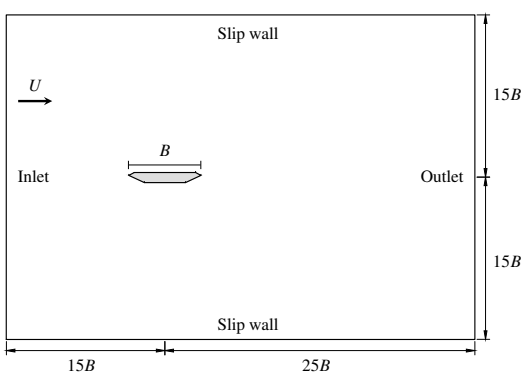

Figure 4: Size of the domain and boundary conditions considered in the CFD analyses .

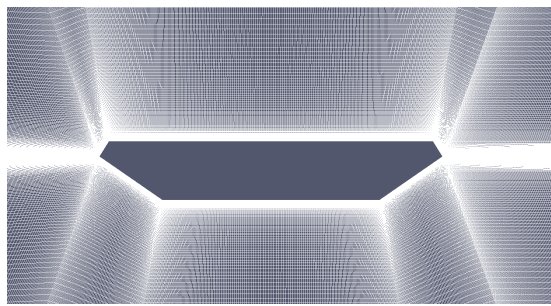

Figure 6: Detail of the mesh of the section $+10 \% \mathrm{H}-10 \% \mathrm{~B}$.

considering a Reynolds number of $10^{5}$, and the walls are modeled using a low Reynolds approach. The boundary conditions considered in the flow domain and its size are shown in figure 4.

The CFD results obtained for three of the samples considered in the surrogate model were validated by means of wind tunnel test conducted at the aerodynamic wind tunnel of the University of La Coruña.

\subsection{Aerodynamic response by means of surrogate modeling}

Although computational power of digital computers and capabilities in the field of CFD are continuously increasing [11], the idea of iterative processes which include CFD evaluations is far for being a suitable alternative in terms of computational time. However, approximations using surrogate models to avoid recursive CFD calculations represents a good alternative to obtain the aerodynamic coefficients of each design requested by the optimization algorithm. The application of surrogate models in the field of multi-objective optimization is tackled for instance, in [17], 
and in the field of optimization in aerodynamics, its application has already started in the area of tall buildings. An interesting application case can be found in [18], where the effects of wind on a building subject to shape modifications by crosssection twisting are studied. More information about surrogate modelling and all the tasks involved in this process are widely described for instance, in [8].

The surrogate model chosen for this work is the Kriging surrogate model, which was first developed in [19], and later on, it was applied to the engineering design in [20]. This technique is nowadays one of the most widespread methods and its performance is widely demonstrated in the literature, for instance, in [21]. In the field of aerodynamics, some applications can be found, as for instance, the work of [22], which employs Kriging surrogate models for optimizing the shape of a building by means of changing the corners of the shape.

The formulation of the Kriging surrogate model can be found for instance, in [8], and can be written as

$$
\hat{f}_{K}(\boldsymbol{x})=\boldsymbol{\kappa}(\boldsymbol{x})^{T} \boldsymbol{\rho}+\varepsilon(\boldsymbol{x}),
$$

where $\boldsymbol{\kappa}(\boldsymbol{x})^{T} \boldsymbol{\rho}$ is a trend function which is combined with a stationary gaussian process error model given by $\varepsilon(\boldsymbol{x})$ that is used to correct the trend function. This stationary gaussian process present a zero mean, constant variance, and the stationary autocorrelation function $r\left(\boldsymbol{x}, \boldsymbol{x}^{\prime}\right)$, in which the most used is the anisotropic generalized exponential model, given by

$$
r\left(\boldsymbol{x}, \boldsymbol{x}^{\prime}\right)=\exp \left(-\sum_{k=1}^{D} \theta_{k}\left|x_{k}-x_{k}^{\prime}\right|^{\gamma}\right) .
$$

The most important task of this process is defining the sampling plan to generate the realization cases for the surrogate model training. The accuracy of the surrogate model is highly conditioned by how these samples represent the full domain. Several techniques to establish a sample plan have been created over the years, mainly based on random generations. Further information about this issue can be found in [8]. In this work, a non-random sample plan based on the authors experience has been carried out, which is shown in figure 7. Vertical and horizontal axes contain the range of variation of $B$ and $H$ with regards to the baseline values of G1 cross-section. The initial design corresponds to the black square and the remaining sample designs are represented by blue circles. It can be observed that the point locations are adequately distributed in the domain considered.

With the values obtained for these designs, a Kriging surrogate model with reduced quadratic trend was built using the Dakota framework [23]. The surrogate model was designed to work with two inputs (the shape parameters $B$ and $H$ ) and six outputs $\left(C_{L}, C_{D}, C_{M}, C_{L}^{\prime}, C_{D}^{\prime}\right.$ and $\left.C_{M}^{\prime}\right)$, and a representation of the outputs with respect to the 2 design variables are shown in figure 9 .

\subsection{Aeroelastic response by means of the quasi-steady theory}

Hence, from the aerodynamic coefficients given by the surrogate model for each pair of shape design variables $(H, B)$, the flutter derivatives of each design can 


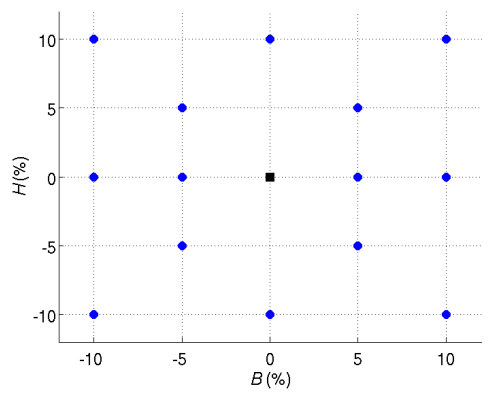

Figure 7: Design of experiments for the surrogate model construction.

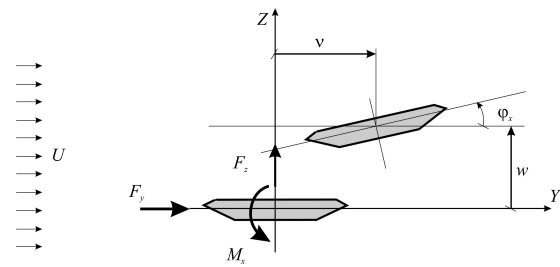

Figure 8: Sign convention for the aeroelastic forces.

be obtained using the quasi-steady theory [10,24], following the sign convention shown in figure 8 and according to the following expressions,

$$
\begin{aligned}
& P_{1}^{*}=-\frac{2 C_{D, 0}}{K} \quad P_{2}^{*}=\frac{C_{D, 0}^{\prime}-C_{L, 0}}{K} \mu_{P} \quad P_{3}^{*}=\frac{C_{D, 0}^{\prime}}{K^{2}} \quad P_{5}^{*}=\frac{C_{D, 0}^{\prime}-C_{L, 0}}{K} \\
& H_{1}^{*}=-\frac{C_{L, 0}^{\prime}+C_{D, 0}}{K} \quad H_{2}^{*}=\frac{C_{L, 0}^{\prime}+C_{D, 0}}{K} \mu_{H} \quad H_{3}^{*}=-\frac{C_{L, 0}^{\prime}}{K^{2}} \quad H_{5}^{*}=-\frac{2 C_{L, 0}}{K} \\
& A_{1}^{*}=\frac{C_{M, 0}^{\prime}}{K} \quad A_{2}^{*}=\frac{C_{M, 0}^{\prime}}{K} \mu_{A} \quad A_{3}^{*}=\frac{C_{M, 0}^{\prime}}{K^{2}} \quad A_{5}^{*}=-\frac{2 C_{M, 0}}{K}
\end{aligned}
$$

where $A_{i}^{*}, H_{i}^{*}$ and $P_{i}^{*}(i=1, \ldots, 6)$ are the flutter derivatives, $C_{L, 0}, C_{M, 0}, C_{D, 0}$, $C_{L, 0}^{\prime}, C_{M, 0}^{\prime}$ and $C_{D, 0}^{\prime}$ are the aerodynamic coefficients and derivatives at zero wind incident angle $\alpha=0$ and $\mu_{P}, \mu_{H}$ and $\mu_{A}$ are the distances between the elastic center of the cross section and the point of application of the aerodynamic forces expressed as a fraction of the width of the section $B$. Once the flutter derivatives are obtained, the flutter velocity of a bridge can be assessed using the Scanlan theory [25] solving the eigenvalue problem considering the aeroelastic forces. The dynamic equilibrium of the bridge is given by

$$
M \ddot{u}+C \dot{u}+K \boldsymbol{u}=\boldsymbol{f}_{a}=\boldsymbol{K}_{a} \boldsymbol{u}+\boldsymbol{C}_{a} \dot{\boldsymbol{u}}
$$

where $\boldsymbol{M}$ is the matrix of masses, $\boldsymbol{C}$ is the damping matrix, $\boldsymbol{K}$ is the stiffness matrix, $\boldsymbol{u}, \dot{\boldsymbol{u}}$ and $\ddot{\boldsymbol{u}}$ represent the vector of displacements, velocities and accelerations, respectively, the subindex $a$ indicates aeroelastic, and $\boldsymbol{f}_{a}$ is the 

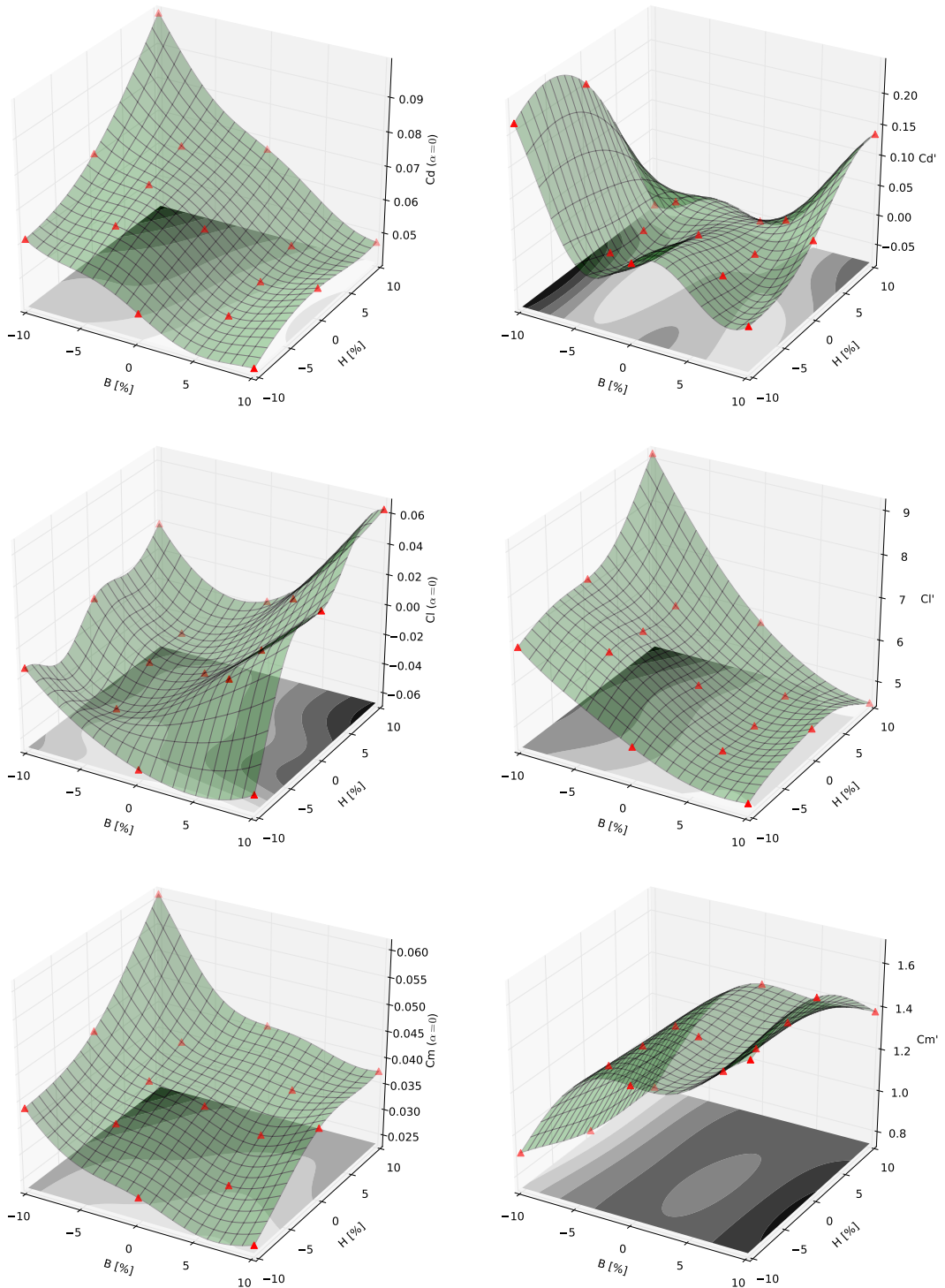

Figure 9: Kriging surrogate model for the aerodynamic coefficients and slopes. 
aeroelastic forces vector, which can be obtained from the flutter derivatives as

$$
\begin{aligned}
\boldsymbol{f}_{a}= & \frac{1}{2} \rho U^{2} K^{2}\left(\begin{array}{ccc}
P_{4}^{*} & -P_{6}^{*} & -B P_{3}^{*} \\
-H_{6}^{*} & H_{4}^{*} & B H_{3}^{*} \\
-B A_{6}^{*} & B A_{4}^{*} & B^{2} A_{3}^{*}
\end{array}\right)\left(\begin{array}{c}
v \\
w \\
\phi_{x}
\end{array}\right) \\
& +\frac{1}{2} \rho U K B\left(\begin{array}{ccc}
P_{1}^{*} & -P_{5}^{*} & -B P_{2}^{*} \\
-H_{5}^{*} & H_{1}^{*} & B H_{2}^{*} \\
-B A_{5}^{*} & B A_{1}^{*} & B^{2} A_{2}^{*}
\end{array}\right)\left(\begin{array}{c}
\dot{v} \\
\dot{w} \\
\dot{\phi}_{x}
\end{array}\right),
\end{aligned}
$$

where $v$ and $w$ are the displacements in the $y$ and $z$ directions, respectively, $\phi_{x}$ are the rotations in the $x$ direction and $K$ represents the reduced frequency, which is given by $K=B \omega / U$ where $\omega$ is the vibration frequency. Further information about this process, and some insights about its implementation can be found in [26].

\section{Approach for the optimum design of cable supported bridges}

The design process of the bridge by means of the surrogate-based optimization approach described in this work consists of two main stages. The first stage requires CFD evaluations of some possible deck cross-sections designs and the construction of a surrogate model that will be recursively evaluated during the optimization process. The second stage is the surrogate-based optimization process, which in each iteration carries out static structural analyses and eigenvalue analyses of the bridge, evaluates the surrogate model, applies the quasi-steady theory and finally solves the eigenvalue problem considering the aeroelastic forces to obtain the flutter velocity. This process can be summarized in the steps listed below.

1. Definition of the lower and upper bounds of the shape design variables.

2. Definition of the sampling plan, establishing the points required for the construction of the surrogate models of the aerodynamic coefficients and slopes for angle of attack $\alpha=0^{\circ}$.

3. Obtaining the aerodynamic coefficients for $\alpha=\left[0^{\circ}, 2^{\circ}\right]$ by means of CFD of the cross-sections proposed in the previous step.

4. Construction of the surrogate model.

5. Definition of the initial values of the design variables. These are the shape and size dimensions of the deck (width $B$, depth $H$ and thickness $t$ ), and the cables cross-section areas and prestressing forces.

6. Optimization process. The stages carried out in each iteration are:

(a) Evaluation of the objective function.

(b) Structural analyses of the static load cases that are included in the design. In this case, five load cases are analyzed, including four load cases representing traffic loads and the self-weight load case of the bridge. These analyses provide the responses for the structural constraints. 
(c) Dynamic analyses of the bridge by means of solving the eigenvalue problem.

(d) Evaluations of the aerodynamic coefficients and slopes, provided by the surrogate model previously built in step 4 .

(e) Evaluations of the flutter derivatives using the quasi-steady theory and the coefficients and slopes obtained in the previous step.

(f) Evaluations of the flutter speed of the bridge by solving the eigenvalue problem having into account the aeroelastic forces, as described in section 2.3. These analyses provide the response of the aeroelastic constraint, which is the flutter velocity of the bridge.

(g) Modification of the design variables.

It must be noticed that the steps (a), (b), (c) and (d) can be run in parallel, while the step (e) has to be carried out from the results of (d), and finally, the step (f) is based on the results of (c) and (e). Besides, the load cases considered in (b) can also be parallelized. This enables a considerable reduction of computing time on each iteration and consequently in the whole optimization process.

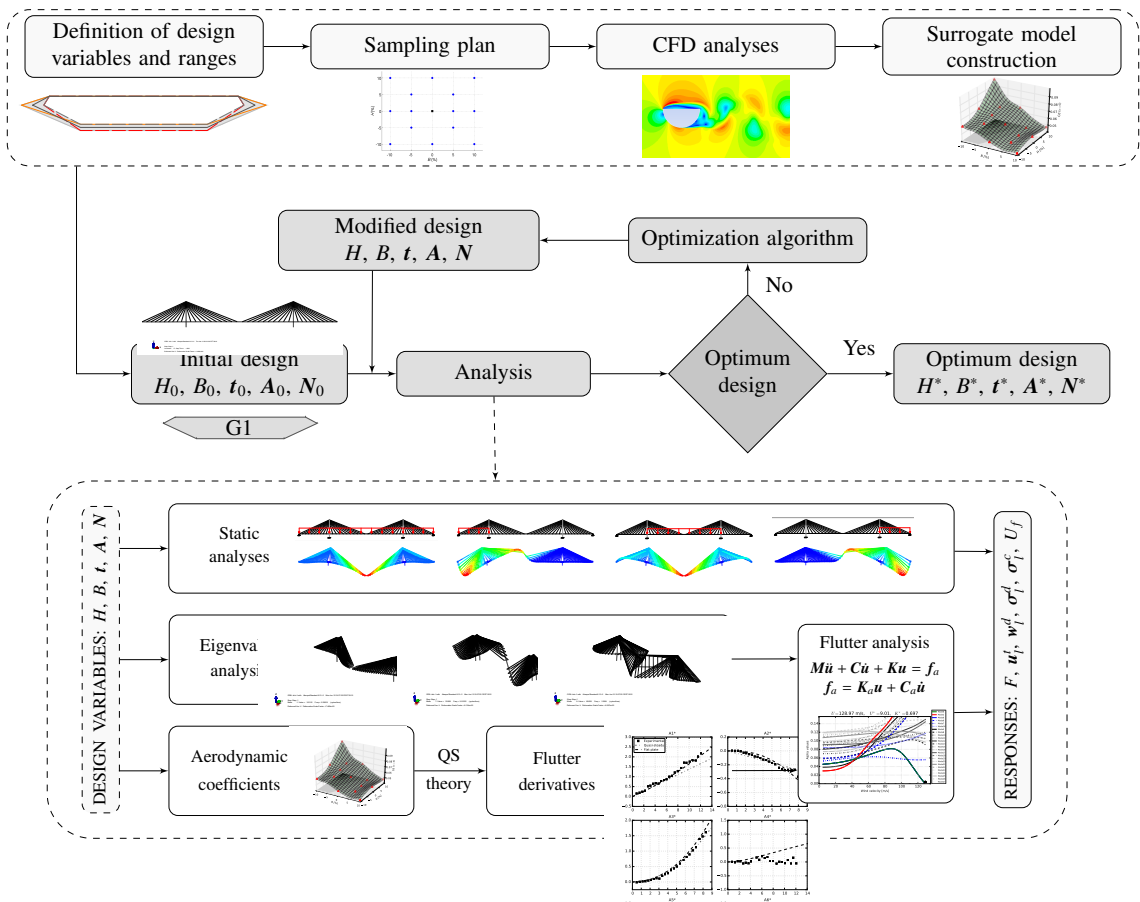

Figure 10: Flowchart of the surrogate-based optimization approach.

This process is also illustrated in the flowchart presented in figure 10, where it can be seen how the surrogate model construction process is carried out before the optimization algorithm starts. Besides, the parallelization capabilities of the 
process are also illustrated in the analysis box, where it can be seen that the static analyses, the eigenvalues analysis and the aerodynamic coefficients evaluation by means of the surrogate model are independent processes and consequently can be carried out simultaneously, allowing the parallel computation of the process. It must be noticed that all the structural static analyses are also independent among them.

The design variables considered in the problem are the deck shape variables $B$ and $H$, the deck plate thickness $t, 40$ cable areas $\boldsymbol{A}$ and 40 prestressing forces $\boldsymbol{N}$. The target of the problem is to minimize the total weight of the bridge. Hence, the objective function is the sum of the volume of deck and cables, and is given by

$$
\min F(H, B, t, \boldsymbol{A}, \boldsymbol{N})=A_{D}(H, B, t) L_{D}+2 \sum_{i=1}^{n} A_{i} L_{C, i}
$$

where $A_{D}$ is the area of the cross-section of the deck, $L_{D}$ is the length of the deck, $n$ is the number of cables of the bridge, which is 40 in this case, $A_{i}$ is the crosssection area of the $i$-esime cable and $L_{C, i}$ its length. The prestressing forces are calculated by being considered as design variables, as explained in [6] or in [4].

Five static load cases are considered: the self-weight of the bridge to assess the prestressing forces of each cable, and four uniform overloads, as shown in figure 10. The structural constraints considered are kinematic and stress constraints for each load case $l$. These are the horizontal displacements of the top of the towers $\boldsymbol{u}_{l}^{t}$, the vertical displacements of the deck in the locations where the stays contact with the deck $\boldsymbol{w}_{l}^{t}$, the stress of each stay of the bridge $\boldsymbol{\sigma}_{l}^{c}$ and the stress in the top and bottom side of the deck in some control points $\sigma_{l}^{d}$, which are 88 response points distributed along the deck. Besides, in order to assess the flutter velocity of each design, a eigenvalues analysis is carried out and the flutter derivatives are obtained employing the quasi-steady theory and the aerodynamic coefficients given by the surrogate model.

Furthermore, all the evaluations carried out in each iteration of the optimization process are also parallelizable, leading to very assumable computational time, which can be about 3 hours for the whole process.

\section{Conclusions}

This paper present some improvements in a methodology previously developed by the authors [6] for the full bridge optimization considering simultaneously structural and aerelastic constraints. The enhancements are related to the aeroelastic characterization of the deck cross-section, and consist of using surrogate models to obtain a more accurate response when the optimization algorithm requires the aerodynamic coefficients of a proposed deck cross-section. After the description of the methodology, some conclusions can be extracted:

- The methodology proposed in [6] can be applied to box type deck, and more complex geometries, increasing the number of deck shape parameters. 
- Application of surrogate models for the aerodynamic characterization of the of the deck cross-section is an accurate and efficient solution, which also allows to predict the errors expected for the responses provided.

- The independence between the processes carried out in the presented methodology allows a high level of parallel computing, leading to reduced computational times which makes the methodology very efficient and competitive.

- This research is currently going on to obtain optimum designs that reduces the total amount of material of the bridge considering structural and aeroelastic constraints simultaneously.

\section{Acknowledgements}

The research leading to these results has received funding from the Spanish Minister of Economy and Competitiveness (MINECO) with reference BIA201341965-P and the Fundación Pedro Barrié de la Maza and the Universidade da Coruña. The authors fully acknowledge the support received.

\section{References}

[1] Larsen, A. \& Wall, A., Shaping of bridge box girders to avoid vortex shedding response. Journal of Wind Engineering and Industrial Aerodynamics, 104-106, pp. 159-165, 2012.

[2] Arora, J.S., Introduction to optimum design. Elsevier/Academic Press, 3rd edition, 2011.

[3] Hernández, S. \& Fontán, A.N., Practical applications of design optimization. WIT Press, 2002.

[4] Baldomir, A., Hernández, S., Nieto, F. \& Jurado, J.A., Cable optimization of a long span cable stayed bridge in La Coruña (Spain). Advances in Engineering Software, 41, pp. 931-938, 2010.

[5] Nieto, F., Hernández, S. \& Jurado, J.A., Optimum design of longspan suspension bridges considering aeroelastic and kinematic constraints. Structural and Multidisciplinary Optimization, 39, pp. 133-151, 2009.

[6] Hernández, S., Cid, M., Nieto, F. \& Jurado, J.A., Optimization of stays and deck shape in cable supported bridges including structural and aeroelastic constrains. BBAA8, 8th International Colloquium on Bluff Body Aerodynamics and Applications, 2016.

[7] Scanlan, R.H. \& Tomko, J.J., Airfoil and bridge deck flutter derivatives. Journal of Engineering Mechanics Division, 97(6), pp. 1717-1737, 1971.

[8] Forrester, A.I.J., Sóbester, A. \& Keane, A.J., Engineering Design via Surrogate Modelling. Wiley, 2008.

[9] Dassault Systèmes, ABAQUS 6.13 Documentation, 2013.

[10] Lazzari, M., Time domain modelling of aerlastic bridge decks: a comparative 
study and an application. International Journal for Numerical Methods in Engineering, 62, pp. 1064-1104, 2005.

[11] Tamura, Y. \& Van Phuc, P., Development of CFD and applications: Monologue by a non-CFD-expert. Journal of Wind Engineering and Industrial Aerodynamics, 144, pp. 3-13, 2015.

[12] Nieto, F., Owen, J.S., Hargreaves, D.M. \& Hernández, S., Bridge deck flutter derivatives: Efficient numerical evaluation exploiting their interdependence. Journal of Wind Engineering and Industrial Aerodynamics, 136, pp. 138150, 2015.

[13] OpenFOAM Foundation Ltd., OpenFOAM User Guide, 2015.

[14] Menter, F.R., Two-equation eddy-viscosity turbulence models for engineering applications. AIAA Journal, 32(8), pp. 1598-1605, 1994.

[15] Menter, F. \& Esch, T., Elements of industrial heat transfer prediction. Sixteenth Brazilian Congress of Mechanical Engineering, 2001.

[16] Nieto, F., Álvarez, A.J., Hernández, S. \& Troche, A., FLUSINI: in-house software for setting up forced oscillation simulations of bridge decks using the OpenFOAM solver. ICWE14, 14th International Conference on Wind Engineering, 2014.

[17] Bouazizi, M.L., Ghanmi, S. \& Bouhaddi, N., Multi-objective optimization in dynamics of the structures with nonlinear behavior: Contributions of the metamodels. Finite Elements in Analysis and Design, 45(10), pp. 612-623, 2009.

[18] Elshaer, A., Bitsuamlak, G. \& El Damatty, A., Aerodynamic shape optimization of tall buildings using twisting and corner modifications. BBAA VIII, 8th International Colloquium on Bluff Body Aerodynamics and Applications, 2016.

[19] Krige, D.G., A statistical approach to some basic mine valuation problems on the witwatersrand. Journal of the Chemical, Metallurgical and Mining Engineering Society of South Africa, 52(6), pp. 119-139, 1951.

[20] Sacks, J., Welch, W.J., Mitchell, T.J. \& Wynn, H., Design and analysis of computer experiments. Statistical Science, 4(4), pp. 409-423, 1989.

[21] Díaz, J., Cid Montoya, M. \& Hernández, S., Efficient methodologies for reliability-based design optimization of composite panels. Advances in Engineering Software, 93, pp. 9-21, 2016.

[22] Bernardini, E., Spence, S.M.J., Wei, D. \& Kareem, A., Aerodynamic shape optimization of civil structures: A CFD-enables Kriging-based approach. Journal of Wind Engineering and Industrial Aerodynamics, 144, pp. 154$164,2015$.

[23] Adams, B., Bauman, L., Bohnhoff, W., Dalbey, K., Ebeida, M., Eddy, J., Eldred, M., Hough, P., Hu, K., Jakeman, J., Swiler, L. \& D., V., DAKOTA, A Multilevel Parallel Object-Oriented Framework for Design Optimization, Parameter Estimation, Uncertainty Quantification, and Sensitivity Analysis: Version 5.3.1 User' Manual. Sandia National Laboratories, December 2009 Updated April 2013.

[24] Febo, S. \& D’Asdia, P., Aerolastic pre-design of stremlined multiple-box 
decks. XI Convegno Nazionale di Ingegneria del Vento IN-VENTO 2010, 2010.

[25] Scanlan, R.H., On flutter and buffeting mechanism in long span bridges. Probabilistic Engineering Mechanics, 3(1), pp. 22-27, 1988.

[26] Jurado, J.Á., Harnández, S., Nieto, F. \& Mosquera, A., Bridge Aeroelasticity: Sensitivity Analysis and Optimal Design. WIT press, 2011. 